\title{
Specific Movement of Esophagus During Transient Lower Esophageal Sphincter Relaxation in Gastroesophageal Reflux Disease
}

\author{
Hoon II Kim, ${ }^{1}$ Su Jin Hong, ${ }^{1 *}$ Jae Pil Han, ${ }^{1}$ Jung Yeon Seo, ${ }^{1}$ Kyoung Hwa Hwang, ${ }^{1}$ Hyo Jin Maeng, ${ }^{1}$ Tae Hee Lee ${ }^{2}$ and Joon \\ Seong Lee ${ }^{2}$ \\ ${ }^{1}$ Department of Internal Medicine, Soonchunhyang University College of Medicine, Bucheon, Gyeonggi-do, Korea; and ${ }^{2}$ Department of \\ Internal Medicine, Soonchunhyang University College of Medicine, Seoul, Korea
}

\section{Background/Aims}

Transient lower esophageal sphincter relaxation (TLESR) is the main mechanism of gastroesophageal reflux disease (GERD). The aim of this study was to investigate the characteristics of transient lower esophageal sphincter movement in patients with or without gastroesophageal reflux by high-resolution manometry (HRM).

\section{Methods}

From June 2010 to July 2010, we enrolled 9 patients with GERD (GERD group) and 9 subjects without GERD (control group), prospectively. The manometry test was performed in a semi-recumbent position for 120 minutes following ingestion of a standardized, mixed liquid and solid meal. HRM was used to identify the frequency and duration of TLESR, esophageal shortening length from incomplete TLESR, upper esophageal sphincter (UES) response, and the related esophageal motor responses during TLESR.

\section{Results}

TLESR occurred in 33 in the GERD group and 34 in the control group after 120 minutes following food ingestion. Duration of TLESR and length of esophageal shortening did not differ between 2 groups. UES pressure increase during TLESR was mostly detected in patients with GERD, and UES relaxation was observed frequently in the control group during TLESR. TLESR-related motor responses terminating in TLESR were predominantly observed in the control group.

\section{Conclusions}

Increased UES pressure was noted frequently in the GERD group, suggesting a mechanism for preventing harmful reflux, which may be composed mainly of fluid on the larynx or pharynx. However, patients with GERD lacked the related motor responses terminating in TLESR to promote esophageal emptying of refluxate.

(J Neurogastroenterol Motil 2013;19:332-337)

Key Words

Gastroesophageal reflux; Manometry; Transient lower esophageal sphincter relaxation

Received: February 12, 2013 Revised: May 7, 2013 Accepted: May 9, 2013

(c) This is an Open Access article distributed under the terms of the Creative Commons Attribution Non-Commercial License (http://creativecommons. org/licenses/by-nc/3.0) which permits unrestricted non-commercial use, distribution, and reproduction in any medium, provided the original work is properly cited.

*Correspondence: Su Jin Hong, MD, PhD

Digestive Disease Center and Research Institute, Soonchunhyang University Bucheon Hospital, 170 Jomaru-ro, Wonmi-gu, Bucheon, Gyeonggi-do 420-767, Korea

Tel: +82-32-621-5087, Fax: +82-32-621-5080, E-mail: sjhong@schmc.ac.kr

Financial support: This work was supported in part by the Soonchunhyang University Research Fund.

Conflicts of interest: None.

Author contributions: Hoon II Kim: writing paper. Su Jin Hong: study design. Jae Pil Han and Jung Yeon Seo: data analysis. Kyung Hwa Hwang and Hyo Jin Maeng: performing procedure. Tae Hee Lee and Joon Seong Lee: review of methods and manuscripts. 


\section{Introduction}

Transient lower esophageal sphincter relaxation (TLESR) is defined as lower esophageal sphincter relaxation that is induced spontaneously without swallowing. TLESR is a physiological mechanism that enables venting of gas from the stomach. ${ }^{1}$ TLESR occurs in patients with gastroesophageal reflux disease (GERD) and in controls. Stomach distension, such as that caused by intragastric air and food, triggers a vasovagal reflex that results in TLESR. This reflex acts as a protective mechanism by preventing the accumulation of excess gas in the stomach or gas from entering the duodenum.

Since Dent et $\mathrm{al}^{2}$ first described TLESR, it has been reported to be the main physiological mechanism in patients with GERD. ${ }^{2-4}$ When TLESR was first identified, it was hypothesized that GERD patients would have a higher rate of TLESR. However, the majority of studies showed a similar rate of TLESR in healthy subjects and in GERD patients. ${ }^{5-8}$ These indicated that acid reflux occurs at a higher frequency during TLESR in patients with GERD than in healthy subjects.'

Many studies have reported the mechanism of TLESR in GERD patients. High-resolution manometry (HRM) has been developed and used to study GERD. HRM is the latest development in esophageal pressure recording. This technique is rapidly gaining recognition, and many expect that it will largely replace conventional manometry. Thus, the advent of HRM will assist investigations of esophageal motor dynamics and the mechanism of TLESR. In this study, we analyzed the characteristics of TLESR and related responses using HRM in GERD patients and a control group.

\section{Materials and Methods}

\section{Patients}

Nine patients with GERD at the Soonchunhyang University Bucheon Hospital from June 2010 to July 2010 were prospectively enrolled in this study. The other nine subjects were registered as healthy individuals and used as controls. Inclusion criteria in the GERD group were: a previous diagnosis of typical reflux symptoms, response to a proton pump inhibitor, and evidence of reflux esophagitis on endoscopic examination. Exclusion criteria were: subjects who had previous gastrointestinal surgery, hiatal hernia, or were taking medications known to affect gastrointestinal motor function or acid secretion. The control group included the healthy volunteers without reflux symptoms and objective endoscopic findings of GERD. The mean age of the GERD group was 51 years and that of the control group was 49 years. The sex ratio was not different between the 2 groups (Table). This study was approved by our institutional review board.

\section{Methods}

The HRM unit used in this study was a solid-state manometric assembly (4.2-mm outer diameter) with 36 circumferential pressure sensors spaced at $1-\mathrm{cm}$ intervals (Sierra Scientific Instruments Inc., Los Angeles, CA, USA). Manometric data were analyzed using the ManoView analysis software (Sierra Scientific Instruments Inc.).

Studies were performed after an at least 8-hour fast, and the manometric assembly was positioned to record from the hypopharynx to the stomach using 5 intragastric sensors. The catheter was fixed in place by taping it to the nose. The manometric protocol included a 5-minute period for assessment of basal sphincter pressure and 10 water swallows of $5 \mathrm{~mL}$ each. Before recording, the pressure transducers were calibrated at 0 and $300 \mathrm{mmHg}$ using externally applied pressure. Patients underwent trans-nasal placement of the manometry assembly, and the assembly was positioned to record from the hypopharynx to the stomach.

Recording was started and continued for 120 minutes after meal ingestion. The standardized, mixed liquid and solid meal consisted of $540 \mathrm{kcal}$ and was composed of rice, sesame porridge, and $200 \mathrm{~mL}$ of whole milk, as suggested by a dietician at our

Table. Comparison of Subject Characteristics and Transient Lower Esophageal Sphincter Relaxation During the 120-minute Postprandial Period in the Gastroesophageal Reflux Disease and Control Groups

\begin{tabular}{lccc}
\hline & $\begin{array}{c}\text { GERD } \\
\text { group }\end{array}$ & $\begin{array}{c}\text { Control } \\
\text { group }\end{array}$ & $P$-value \\
\hline No. of subjects & 9 & 9 & \\
Mean age (yr) & 51 & 49 & $\mathrm{NS}$ \\
Male (n [\%]) & $4(44.4)$ & $5(55.5)$ & $\mathrm{NS}$ \\
Total No. of TLESR events & 33 & 34 & $\mathrm{NS}$ \\
Complete TLESR & 7 & 8 & \\
Incomplete TLESR & 26 & 26 & \\
Duration of TLESR (sec) & $16.1 \pm 3.9$ & $17.9 \pm 7.0$ & $\mathrm{NS}$ \\
Length of ES (mm) & $10.2 \pm 7.1$ & $9.8 \pm 10.2$ & $\mathrm{NS}$ \\
\hline
\end{tabular}

GERD, gastroesophageal reflux disease; TLESR, transient lower esophageal sphincter relaxation; ES, esophageal shortening; NS, not significant. 
hospital. Following meal ingestion, the subjects were situated in a semi-recumbent position and were requested to minimize head movements.

TLESR was defined according to previously published criteria adapted for HRM. ${ }^{10}$ TLESR was identified as follows: absence of swallowing from 4 seconds before to 2 seconds after the onset of LES relaxation, LES relaxation rate of $\geq 1 \mathrm{mmHg} / \mathrm{sec}$, a time from the onset to complete relaxation of $\leq 10$ seconds, and a nadir pressure $<2 \mathrm{mmHg}$. LES relaxations that lasted for more than 10 seconds and that were associated with a swallow within 5 seconds before or 2 seconds after the onset of LES relaxation were also included as TLESRs. According to the modified criteria, inhibition of the crural diaphragm, prominent LES after-contraction, and the presence of esophageal common cavity were accepted as additional criteria. ${ }^{10}$

LES and esophageal pressure recordings were displayed as HRM color plots. TLESR was classified as complete and incomplete types which were described by Mittal et al. ${ }^{11}$ Complete TLESR was defined as an end-expiratory LES pressure of $<2$ $\mathrm{mmHg}$ at the peak of relaxation. Incomplete TLESR was defined as an end-expiratory LES pressure of $>5 \mathrm{mmHg}$ during relaxation. ${ }^{11}$ Esophageal shortening length during TLESR was calculated by LES lift estimated for incomplete TLESR (Fig. 1). The esophageal common cavity was defined as a sharp and sustained rise in end-expiratory esophageal pressure of $\geq 4 \mathrm{mmHg}$ in association with TLESR. ${ }^{12}$

The upper esophageal sphincter (UES) pressure during TLESR was monitored by HRM. The UES responses were classified as a UES pressure increase, a UES pressure decrease, and UES relaxation. A UES pressure increase was considered when the UES pressure increased by $\geq 10 \mathrm{mmHg}$ from the baseline UES pressure. ${ }^{13,14}$ A UES pressure decrease was defined when the UES pressure decreased by $\leq 10 \mathrm{mmHg}$ from the baseline UES pressure. UES relaxation was identified as a rapid drop of at least $10 \mathrm{mmHg}$ in the UES pressure only if not accompanied by primary peristalsis. ${ }^{13}$ UES relaxation was defined as complete if the nadir pressure was $<5 \mathrm{mmHg}$ of the proximal esophageal pressure. TLESR-terminating esophageal motor events were identified in each group as (1) primary peristalsis (PP), defined as a full propagating wave from UES to LES swallow-related UES relaxation and pharyngeal contraction; (2) full secondary contraction (FSC), defined as a full propagating wave form of UES to LES without UES relaxation and pharyngeal contraction; and (3) partial secondary contraction (PSC), defined as a secondary contraction wave originating below the level of the UES. ${ }^{15}$

\section{Statistical Methods}

Between the 2 groups, categorical variables were assessed using the Chi-square test or Fisher's exact test, and noncategorical variables were compared using Wilcoxon's rank-sum test. The frequency and duration of TLESR, extent of esophageal shortening, and frequency of UES relaxation were summarized using medians and 5 th-95th percentile ranges or means \pm standard deviations, unless otherwise indicated. A $P$-value $<0.05$ was considered to indicate significance.

\section{Results}

\section{Transient Lower Esophageal Sphincter Relaxation Characteristics}

Manometry was well-tolerated by all 18 subjects, and they had normal deglutitive esophagogastric junction (EGJ) relaxation. We recorded and analyzed 67 TLESR events with a median of 3.5 (range, 0-13) events per subject during the 120-minute postprandial period. The frequency of TLESR was 33 in the GERD group and 34 in the control group. The median number
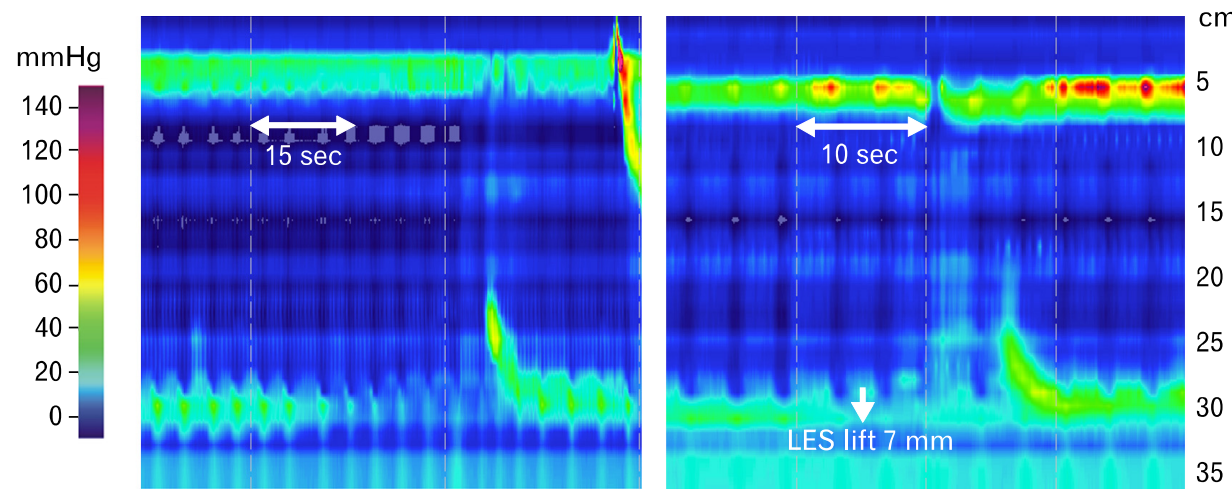

Figure 1. Transient lower esophageal sphincter relaxation (TLESR) events. Left panel indicates complete TLESR terminated by partial secondary contractions. Right panel shows incomplete TLESR terminated by partial secondary contractions. The length of esophageal shortening by lower esophageal sphincter (LES) lift during incomplete relaxation was calculated to be $7 \mathrm{~mm}$. 
of TLESR events was not significantly higher in the GERD group $(\mathrm{n}=3.5)$ than those in the control group $(\mathrm{n}=3.6)$. Incomplete TLESR was more common than complete TLESR in both groups. Fifty-two incomplete TLESR events were analyzed, and the ratio of incomplete TLESR to total TLESR events was not different between the 2 groups. No difference in the mean duration of TLESR or the mean length of esophageal shortening was observed between the 2 groups. The median duration of a TLESR event was $16.1 \pm 3.9$ seconds in the GERD group and $17.9 \pm 7.0$ seconds in the control group $(P=0.863)$. The mean length of esophageal shortening was $10.2 \pm 7.1 \mathrm{~mm}$ in the GERD group and $9.8 \pm 10.2 \mathrm{~mm}$ in the control group $(P=$ 0.372) (Table).

\section{Basal Upper Esophageal Sphincter Pressure Change and Upper Esophageal Sphincter Relaxation During Transient Lower Esophageal Sphincter Relaxation}

Patients with GERD showed primarily increasing UES pressure during TLESR. Twenty-five TLESR (75.8\%) events revealed increasing UES pressure in the GERD group. However, only six were associated with increasing UES pressure in the control group. The frequency of increasing UES pressure differed between the groups $(P=0.001)$ (Fig. 2). The percentages of UES relaxation were $21.2 \%$ in the GERD group and $73.5 \%$ in the control group $(P=0.001)$.

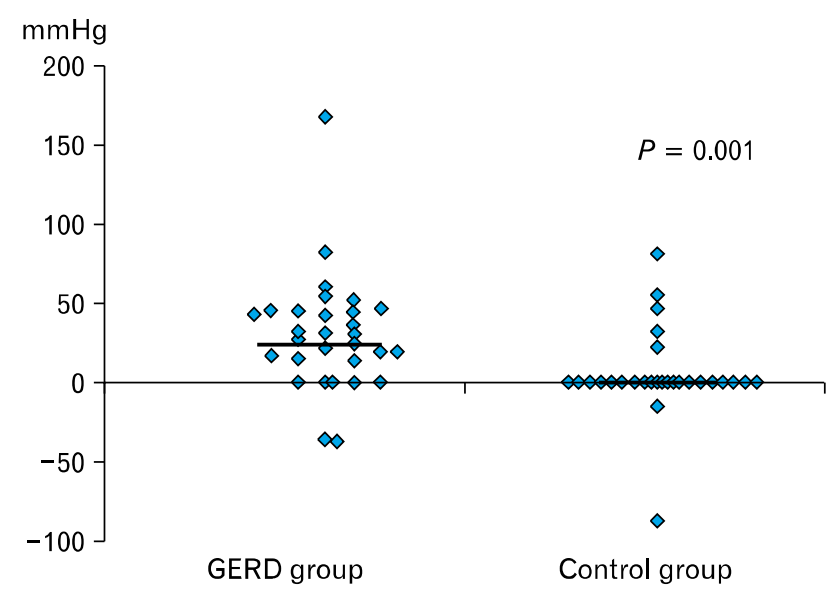

Figure 2. Upper esophageal sphincter (UES) pressure changes during transient lower esophageal sphincter relaxation (TLESR). Most patients with gastroesophageal reflux show an increase in UES pressure during TLESR. However, many controls exhibit no UES pressure changes during TLESR. GERD, gastroesophageal reflux disease.

\section{Transient Lower Esophageal Sphincter Relaxation Terminating Esophageal Motor Events}

PSC was the major motor activity that terminated TLESR in both groups. The percentages of TLESR events terminated by PP, FSC and PSC were $24.2 \%, 3.0 \%$ and $45.5 \%$ in the GERD group, respectively. The percentages of TLESR events terminated by PP, FSC and PSC in the control group were $44.1 \%$, $17.6 \%$ and $35.3 \%$, respectively. About $27.3 \%$ of TLESR events did not accompany motor events in the GERD group, compared with $2.9 \%$ in the control group $(P=0.005)$.

\section{Discussion}

TLESR events comprise the main mechanism leading to acid reflux and are responsible for $70 \%$ of acid-reflux episodes. $^{2,12,16-19}$ Since GERD is characterized by reflux, TLESR plays an important role in the etiology of this condition.

HRM is a relatively new technique that allows visualization of esophageal pressure and identification of TLESR. HRM uses a large number of closely placed solid-state pressure transducers so that intraluminal pressure can be monitored as a continuum, much as time is viewed as a continuum on line tracings during conventional manometry. HRM has several advantages compared with conventional manometry. HRM is simpler to set up and has improved sphincter localization; moreover, movement of the catheter relative to the LES does not impair data quality. Additionally, HRM allows a more detailed view of pressure in the LES, esophageal body and UES. Furthermore, HRM can be interpreted simply and requires a shorter procedural time than conventional manometry. Thus, HRM will replace conventional manometry utilizing a line-tracing format. HRM is more efficient than conventional manometry for detection and analysis of TLESR. It provides increased sensitivity by the almost continuous monitoring through the pressure transducers. Therefore, it can decrease the artifacts resulted from probe movement or esophageal shortening. ${ }^{20,21}$

When TLESR was first identified, it was expected that GERD patients would have a higher rate of TLESR. However, the majority of studies report a similar rate of TLESR in healthy subjects and GERD patients. ${ }^{5-8}$ Schneider et $\mathrm{al}^{22}$ reported that the frequency of TLESR was more than twice as high in patients with GERD compared to healthy control group. ${ }^{22}$ Thus, there is debate about whether the frequency of TLESR is increased in 
patients with GERD. To-date, most studies of TLESR frequency used conventional manometry; few used HRM. Thus, it is meaningful that we examined TLESR using HRM. In our study, the frequency of TLESR in the GERD group was similar to that in the control group.

In contrast, several studies have reported that TLESR in patients with GERD is more correlated with acid reflux than that in the normal population. This means that a higher percentage of TLESR events is associated with gastroesophageal reflux in patients with GERD. ${ }^{9}$ Because we did not monitor $\mathrm{pH}$ during this study, we did not assess the amount of acid reflux during TLESR.

TLESR is triggered by gastric distension, which induces an autonomic reflex. Some investigators have reported that contraction of the distal esophageal longitudinal muscle occurs during TLESR. ${ }^{23-25}$ So, longitudinal muscle contraction is a key event during TLESR. In 2006, Pandolfino et $\mathrm{al}^{24}$ reported a TLESR study using HRM and radio-opaque clips. That study showed that the key events leading to EGJ opening during TLESR were LES relaxation, crural diaphragm inhibition, esophageal shortening, and a positive pressure gradient between the stomach and the EGJ lumen. Similar to the present study, esophageal longitudinal muscle contraction (LMC) occurred after the onset of LES relaxation and was correlated with acid reflux. ${ }^{24}$ However, in 2010, Schneider et al ${ }^{22}$ reported that esophageal LMC is restricted to the distal esophagus, begins before TLESR onset, and is sustained during the entire TLESR duration. The authors proposed that LMC of the esophagus may be the primary motor event, and that the LES and crural diaphragm could be secondary. ${ }^{22}$ This result was significant because it suggested for the first time that LMC could be used to develop new medications for GERD.

Mittal et $\mathrm{al}^{11}$ reported a feasibility study in which LES lift seen on HRM was a possible surrogate marker for LMC. Their findings indicated that LES lift during incomplete TLESR suggests a possible cause and effect relationship between LMC and LES relaxation. They classified TLESR as complete and incomplete types. Complete TLESR was defined when the residual LES pressure was $<2 \mathrm{mmHg}$ at the peak of relaxation, and incomplete TLESR was defined when the residual LES pressure during relaxation was $>5 \mathrm{mmHg}$. During complete TLESR, LES lift could not be measured, because the LES was completely relaxed. However, LES lift could be measured during incomplete TLESR. Although it was significant that LMC could be measured only by HRM, there was a difference in the degree of
LES relaxation and LES lift between patients with GERD and a healthy control group.

Thus, we hypothesize that the esophageal motor responses and LMC during TLESR in patients with GERD may be different compared to those in the healthy population. We compared the length of esophageal longitudinal muscle shortening by measuring LES lift during incomplete TLESR in both GERD patients and controls. Although this is not a direct method of LMC estimation, we analyzed our manometric data under the precondition that LES lift reflects LMC on HRM. ${ }^{11}$ No difference in the degree of shortening of esophageal longitudinal muscles was identified in the GERD and control groups. Our data also suggest that the mean duration of TLESR was not different between the GERD and control groups.

However, the differences in UES pressure change, UES relaxation, and related motor responses during TLESR explain esophageal dynamics in subjects with and without GERD. We assume that the UES pressure increase and infrequent UES relaxation in the GERD group resulted from GERD itself, and that the two UES responses were secondary motor responses due to the more acidic fluid refluxate. ${ }^{26}$ These motor actions probably protect the larynx, pharynx and lung from the harmful refluxate. $^{14}$

TLESR usually terminates by esophageal contractions, including PP and secondary contractions. ${ }^{15}$ Such esophageal contractions promote fast esophageal emptying of refluxate into the stomach. In our study, patients with GERD had a relatively high proportion of TLESR without later esophageal contraction compared with that of controls. Delayed esophageal emptying of refluxate may worsen the esophageal environment after gastroesophageal reflux. Extended refluxate contact time induces esophageal mucosal damage.

Our study had several limitations. First, it was observational and included a small number of patients. Second, LES lift was estimated only for incomplete TLESR. We were unable to calculate the height of esophageal shortening during complete TLESR. However, complete TLESR may be more important than incomplete TLESR in the pathogenesis of GERD. Third, we did not consider GERD severity. Fourth, our testing was performed using only HRM and did not include other tools for estimating esophageal motor and biological functions.

In conclusion, TLESR events did not differ between the GERD and control groups. UES responses after TLESR were reasonably regulated in patients with GERD. However, the paucity of TLESR-related esophageal contractions may be a con- 
cern in terms of enhanced mucosal damages in GERD patients.

\section{Acknowledgements}

We thank the nursing staff for their support and assistance with the procedures.

\section{References}

1. Wyman JB, Dent J, Heddle R, Dodds WJ, Toouli J, Downton J. Control of belching by the lower oesophageal sphincter. Gut 1990; 31:639-646.

2. Dent J, Dodds WJ, Friedman RH, et al. Mechanism of gastroesophageal reflux in recumbent asymptomatic human subjects. J Clin Invest 1980;65:256-267.

3. Massey BT, Simuncak C, LeCapitaine-Dana NJ, Pudur S. Transient lower esophageal sphincter relaxations do not result from passive opening of the cardia by gastric distention. Gastroenterology 2006;130:89-95.

4. Holloway RH, Hongo M, Berger K, McCallum RW. Gastric distention: a mechanism for postprandial gastroesophageal reflux. Gastroenterology 1985;89:779-784.

5. Iwakiri K, Hayashi Y, Kotoyori M, et al. Transient lower esophageal sphincter relaxations (TLESRs) are the major mechanism of gastroesophageal reflux but are not the cause of reflux disease. Dig Dis Sci 2005;50:1072-1077.

6. Trudgill NJ, Riley SA. Transient lower esophageal sphincter relaxations are no more frequent in patients with gastroesophageal reflux disease than in asymptomatic volunteers. Am J Gastroenterol 2001; 96:2569-2574.

7. Sifrim D, Holloway R. Transient lower esophageal sphincter relaxations: how many or how harmful? Am J Gastroenterol 2001;96: 2529-2532.

8. Grossi L, Ciccaglione AF, Travaglini N, Marzio L. Transient lower esophageal sphincter relaxations and gastroesophageal reflux episodes in healthy subjects and GERD patients during 24 hours. Dig Dis Sci 2001;46:815-821.

9. Bredenoord AJ, Weusten BL, Timmer R, Smout AJ. Gastro-oesophageal reflux of liquids and gas during transient lower oesophageal sphincter relaxations. Neurogastroenterol Motil 2006;18:888-893.

10. Holloway RH, Penagini R, Ireland AC. Criteria for objective definition of transient lower esophageal sphincter relaxation. Am J Physiol 1995;268(1 Pt 1):G128-G133.

11. Mittal RK, Karstens A, Leslie E, Babaei A, Bhargava V. Ambulatory high-resolution manometry, lower esophageal sphincter lift and transient lower esophageal sphincter relaxation. Neurogastroenterol Motil 2012;24:40-46, e2.

12. Mittal RK, Holloway RH, Penagini R, Blackshaw LA, Dent J. Transient lower esophageal sphincter relaxation. Gastroenterology 1995;109:601-610.

13. Pandolfino JE, Ghosh SK, Zhang Q, Han A, Kahrilas PJ. Upper sphincter function during transient lower oesophageal sphincter relaxation (tLOSR); it is mainly about microburps. Neurogastroenterol Motil 2007;19:203-210.

14. Torrico S, Kern M, Aslam M, et al. Upper esophageal sphincter function during gastroesophageal reflux events revisited. Am J Physiol Gastrointest Liver Physiol 2000;279:G262-G267.

15. Kuribayashi S, Massey BT, Hafeezullah M, et al. Terminating motor events for TLESR are influenced by the presence and distribution of refluxate. Am J Physiol Gastrointest Liver Physiol 2009;297: G71-G75.

16. Penagini R, Schoeman MN, Dent J, Tippett MD, Holloway RH. Motor events underlying gastro-oesophageal reflux in ambulant patients with reflux oesophagitis. Neurogastroenterol Motil 1996;8: 131-141.

17. Schoeman MN, Tippett MD, Akkermans LM, Dent J, Holloway RH. Mechanisms of gastroesophageal reflux in ambulant healthy human subjects. Gastroenterology 1995;108:83-91.

18. Mittal RK, McCallum RW. Characteristics and frequency of transient relaxations of the lower esophageal sphincter in patients with reflux esophagitis. Gastroenterology 1988;95:593-599.

19. Dodds WJ, Dent J, Hogan WJ, et al. Mechanisms of gastroesophageal reflux in patients with reflux esophagitis. N Engl J Med 1982;307:1547-1552.

20. Roman S, Zerbib F, Belhocine K, des Varannes SB, Mion F. High resolution manometry to detect transient lower oesophageal sphincter relaxations: diagnostic accuracy compared with perfused-sleeve manometry, and the definition of new detection criteria. Aliment Pharmacol Ther 2011;34:384-393.

21. Rohof WO, Boeckxstaens GE, Hirsch DP. High-resolution esophageal pressure topography is superior to conventional sleeve manometry for the detection of transient lower esophageal sphincter relaxations associated with a reflux event. Neurogastroenterol Motil 2011;23:427-432, e173.

22. Schneider JH, Küper MA, Königsrainer A, Brücher BL. Transient lower esophageal sphincter relaxation and esophageal motor response. J Surg Res 2010;159:714-719.

23. Babaei A, Bhargava V, Korsapati H, Zheng WH, Mittal RK. A unique longitudinal muscle contraction pattern associated with transient lower esophageal sphincter relaxation. Gastroenterology 2008; 134:1322-1331.

24. Pandolfino JE, Zhang QG, Ghosh SK, Han A, Boniquit C, Kahrilas PJ. Transient lower esophageal sphincter relaxations and reflux: mechanistic analysis using concurrent fluoroscopy and high-resolution manometry. Gastroenterology 2006;131:1725-1733.

25. Shi G, Pandolfino JE, Joehl RJ, Brasseur JG, Kahrilas PJ. Distinct patterns of oesophageal shortening during primary peristalsis, secondary peristalsis and transient lower oesophageal sphincter relaxation. Neurogastroenterol Motil 2002;14:505-512.

26. Babaei A, Bhargava V, Mittal RK. Upper esophageal sphincter during transient lower esophageal sphincter relaxation: effects of reflux content and posture. Am J Physiol Gastrointest Liver Physiol 2010; 298:G601-G607. 